\title{
The effect of mobile phone electromagnetic radiation on brain vessels
}

\author{
M Bakhmetev AS ${ }^{1}$, Kaliaev $\mathrm{AO}^{2}$, Sukhoruchkin $\mathrm{AA}^{3}$ and Malikova $\mathrm{MA}^{4}$ \\ ${ }^{1}$ Saratov State Medical University named after V. I. Razumovsky, Saratov, Russia \\ ${ }^{2}$ Avesta Central Hospital, Saratov, Russia \\ ${ }^{3}$ Stroygrad Medical Center, Kirsanov, Russia. \\ ${ }^{4}$ Boston University, Boston Medical Center, Department of Surgery, Boston, MA, USA 02181
}

\begin{abstract}
Objective : This is a study to assess the impact of the global system for mobile communication (GSM) mobile phone electromagnetic radiation on the main vessels of the brain.

Material and methods: This was a cross sectional study which included 55 volunteers between 18-66 years old, who were examined by stationary device Philips HD 11 expert class phased sectoral sensor with a frequency of $2-4 \mathrm{MHz}$ at the Ultrasonic and Functional Diagnostics Department of the Mirotvortseva hospital, which is affiliated with Saratov State Medical University (SSMU) and in the clinic "Stroygrad Medical Center " in Kirsanov city, Tambov region.

Results: The study included 55 volunteers between 18-66 years old (average age was 38.95 years) with permeable trans-temporal acoustic windows and without chronic diseases such as hypertension, diabetes mellitus, chronic cerebral ischemia, coronary heart disease and no initial spasm in middle cerebral artery (MCA). The study was conducted in the supine position with enabled mobile phone in talk mode for 7 minutes. The blood flow velocity (BFV) and resistive index (RI) in the MCA (middle cerebral artery) were measured on both sides at baseline and after 7 minutes of talking on a mobile phone. Cerebral vasospasm was considered if detected RI was 0.65 and above.

In order to minimize the measurement error, the same modern mobile phone was utilized for all studied subjects. The analysis of the data revealed an initial increase in the BFV and RI in the MCA in all patients by the end of 7 minutes conversation. Unilateral spasm of various degree was detected by ultrasound in the middle cerebral artery of 27 out of 55 examined patients (50\%) after 7 minutes of them talking on a mobile phone. Average RI value was $0.66 \pm 0.04$ on the ipsilateral side of MCA with range of a minimum RI detected at 0.59 , and maximum value of 0.75 . On the contralateral side of MCA an average RI value was detected as $0.66 \pm$ 0.04 , with range of $0.60-0.74$. Therefore, interaction with mobile phone for period of time up to 7 minutes in most cases gave rise to moderately expressed spasm on both sides. The results were independent of gender and blood pressure.
\end{abstract}

Conclusions: MCA spasm recorded as a result of prolonged mobile phone call (about 7 minutes), was detected in $60 \%$ of examined subjects, which may indicate the importance of this potential risk factor in causing headaches and other negative psychological factors (i.e. lack of attention, sleep disturbances, irritability etc.).

\section{Introduction}

The 21st century is the time of high level technology. There are a lot of gadgets of telecommunications in everyday use. The most popular gadgets are cell or mobile phones, and smartphones. The dramatic worldwide increase in use of cellular phones has prompted concerns regarding potential harmful effects of exposure to radiofrequencymodulated electromagnetic fields (RF-EMFs). Of a particular concern has been the potential carcinogenic effects from the RF-EMF emissions of cell phones. [1] However, the results of epidemiologic studies, which examined association between the cell phone use and prevalence of brain tumors, have been inconsistent. Some studies showed increased risk, but the issue remains unresolved. [2]. RF-EMFs emitted by cell phones are absorbed in the brain within a range that could influence neuronal activity. [3] Thermal effects from RF-EMFs have also been invoked as a mechanism that could affect neuronal activity, although temperature changes produced by current cell phone technology are likely minimal. [4] Numerous studies have examined the effect of RF-EMR on a persons' psychological status, the correlation between the duration of a call, and the development of cancer disease, etc. We found no results in current publications that demonstrated RF-EMR effect on brain vessels. It is well-established fact that persistent cerebral vasospasm causes persistent headaches, reduces efficiency, causes irritation, fatigue and disturbed sleep patterns. [5-8]

\section{Material and methods}

This was a cross sectional study which included 55 volunteers between 18-66 years old with permeable trans temporal acoustic windows, who had no chronic diseases such as hypertension, diabetes mellitus, chronic cerebral ischemia, coronary heart disease. Patients with atherosclerotic diseases of brachiocephalic arteries which were complicated with stenosis, as well as with spasm of the middle cerebral

Correspondence to: Malikova MA, Executive Director, Surgical Translational Research Operations and Compliance Department of Surgery, Boston Medical Center, Boston University 88 East Newton Street Collamore Building, Suite D507-A Boston, MA 02118, USA, Tel: (617) 414-6836; E-mail: mmalikov@bu.edu

Key words: radiofrequency-modulated electromagnetic fields, cerebral vasospasm.

Received: November 20, 2017; Accepted: December 18, 2017; Published: December 22, 2017 


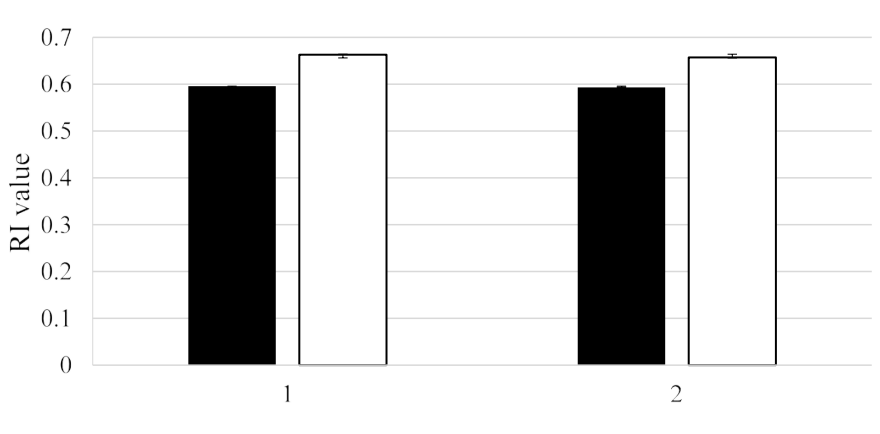

- At Baseline $\square$ After mobile phone interaction for 7 minutes

Figure 1. Average Resistive index (RI) on the ipsilateral (1) and contralateral (2) sides of middle cerebral artery (MCA) before and after effect of the electromagnetic radiation (EMR) emitted by the mobile phone. Average RI value after mobile phone interaction was $0.66 \pm 0.04$ on the ipsilateral side of MCA with range of a minimum RI detected at 0.59 , and maximum value of 0.75 . On the contralateral side of the MCA an average RI value was $0.66 \pm 0.04$, with range $0.60-0.74$ detected after mobile phone interaction for 7 minutes.

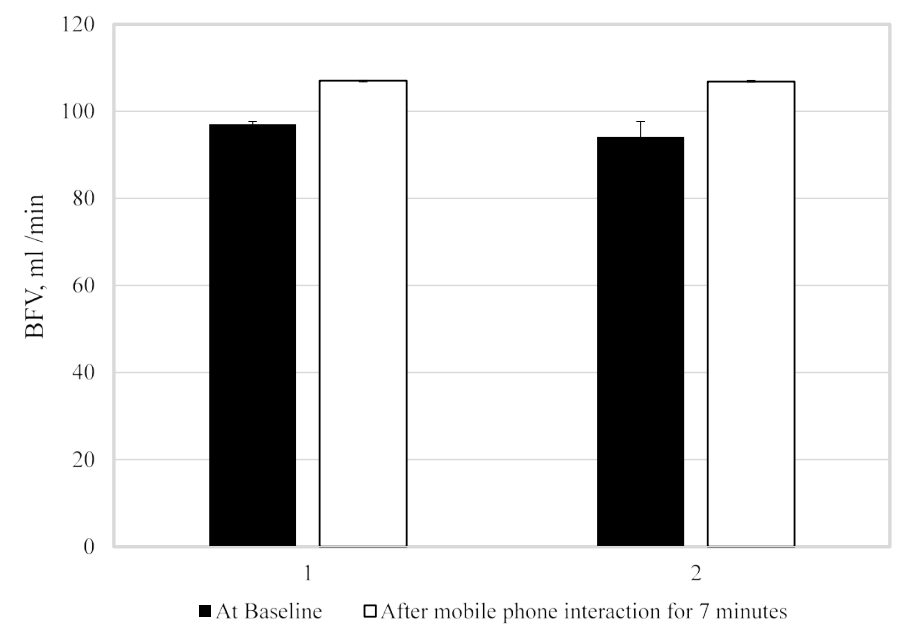

Figure 2. Blood flow velocity (BFV). Average BFV on the ipsilateral (1) and contralateral (2) sides of middle cerebral artery (MCA) before and after the effect of the electromagnetic radiation (EMR) emitted by the mobile phone. The blood flow velocity (BFV) average value measured at baseline was $97.04 \pm 18.19$ versus $107.05 \pm 18.87$ post mobile phone interaction on ipsilateral side; and $94.11 \pm 14.79$ versus $106.84 \pm 14.52$, respectively on the contralateral side of MCA.

artery (MCA) and posterior cerebral artery (PCA) were excluded from the study. In accordance with Declaration of Helsinki, Institutional Review Board (IRB) approval and informed consents from each individual subject were obtained prior to any study procedures were performed. Written informed consent was obtained prior to the experiment and a specialized ethical committee of the Saratov State Medical University for research on human subjects approved the study protocol.

The study was carried out in the supine position with the mobile phone held on the right side of their head, which was in the talk mode for 7 minutes. Volunteers were instructed to abstain from caffeine, alcohol and medication, keep a regular sleep-wake schedule (8 hours sleep starting not later than midnight on the day prior to the study), and specific instructions do not use mobile phone 2 hours prior to the study.

The study was performed by stationary device Philips HD 11 expert class phased sectoral sensor with a frequency of $2-4 \mathrm{MHz}$ at the Ultrasonic and Functional Diagnostics Department of the Mirotvortseva hospital, which is affiliated with Saratov State Medical University (SSMU) and in the clinic "Stroygrad Medical Center" in
Kirsanov city of Tambov region on the device of expert class Mindray DC-8 phased sensor with a frequency of 2-4 MHz. The cell phone had a GSM global system for mobile communication (GSM) about $900 \mathrm{MHz}$ chip. We measured blood flow velocity (BFV) and resistive index (RI) in the MCA on both sides.

The data obtained from 55 subjects were analyzed using SAS program, version V 9.1 (SAS Institute Inc., Cary, NC, USA).

\section{Results}

Subjects were considered to have cerebral vasospasm if RI of 0.65 and above was detected. The RI with mildly expressed spasm of middle cerebral artery (MCA) fluctuated in the range of $0.65-0.69$, with moderately expressed spasm from RI detected at 0.7 to 0.74 and with severe spasm of more than 0.75 . Both BFV and RI on both sides of MCA were measured before mobile phone exposure (baseline), and then after 7 minutes of talking on the phone. In order to minimize measurement error we used the same modern cell phone for all volunteers. In the analysis of the data we found an increase in the BFV and RI in the MCA consistently in all patients by the end of first minute of conversation. By the expiration of 7 minutes an ultrasonic sign of spasm of the middle cerebral artery were detected in ipsilateral side of 30 patients out of 55 patients examined $(60 \%)$, and in contralateral side of 22 patients (40\%). Average RI value after interaction with mobile phone for 7 minutes was $0.66 \pm 0.04$ on the ipsilateral side of MCA with range of a minimum RI detected at 0.59 , and maximum value of 0.75 . On the contralateral side of MCA an average RI value was $0.66 \pm 0.04$, with range $0.60-0.74$ detected. The difference in RI values detected in the MCA at the baseline examination and those measured after effect of the electromagnetic radiation (EMR) emitted by the mobile phone were highly statistically significant

$\left(\mathrm{P}=1.4354 \times 10^{-20}\right.$ for ipsilateral and $\mathrm{P}=3.1407 \times 10^{-17}$ for contralateral sides of MCA, respectively).

Therefore, interaction with mobile phone for period of time up to 7 minutes in most cases gave rise to moderately expressed spasm on both sides. The results were independent of gender and blood pressure.

The blood flow velocity (BFV) average value measured at baseline was $97.04 \pm 18.19$ versus $107.05 \pm 18.87$ on ipsilateral side; and $94.11 \pm 14.79$ versus $106.84 \pm 14.52$ on the contralateral side of MCA. The difference in BFV found between the baseline and post EMR administration for 7 minutes were statistically significant $\left(\mathrm{P}=8.21045 \times 10^{-9}\right.$ on ipsilateral and $\mathrm{P}=2.4639 \times 10^{-8}$ on contralateral sides of the MCA, respectively).

\section{Discussion}

When analyzing the data obtained, an increase in BFV and RI in the MCA was found in all 55 examined subjects (100\%) after 7 minutes of exposure to electromagnetic radiation emitted by mobile phone device.

The spasm of various degree was detected bilaterally in the MCA in 22 examined subjects by the end of a long conversation on a mobile phone $(40 \%)$. The moderately expressed unilateral spasm (mean RI value 0.72 ) was observed in 11 subjects out of 55 studied in total (20 $\%)$, including men with average age of 23.3 and women with average age 36.6 years old. We noted a trend that patients who were 45 years old and above were less affected by EMR. This observation can be attributed to the more rigid arterial walls in older patients.

The spasm with mild to severe degree was detected in the younger group of subjects (18-45 years old). The artery walls are more elastic 
and probably more prone to be affected by EMR at a younger age. Thus, ultrasound exam allowed to see changes in the brain artery in majority of patients bilaterally after 7 minutes of interaction with EMR emitted by the mobile phone.

\section{Conclusions}

In this pilot study the talk for more than 7 minutes on a cell phone in most cases $(60 \%)$ led to the spasm of the main cerebral artery in the assessed subjects, which indicates the high importance of EMR as a potential risk factor for headaches and other negative psychological factors (i.e. lack of attention, disturbance of the structure of sleep, irritability, memory impairment, etc.). When comparing the occurrence of spasm between ipsilateral and contralateral sides of MCA, it was noted that in most cases the ipsilateral side of the MCA got affected mostly. However, in some patients unilateral changes of RI and BFV were observed in either ipsilateral or contralateral side of the MCA. This is a pilot study which requires further prospective, randomized, controlled investigations to fully understand the degree of the effect of electromagnetic radiation emitted by the cell phones on the blood vessels of the brain.

\section{References}

1. Volkow N.D, Tomasi D, Wang G.J, Vaska P, Joanna S. Fowler J.S (2011) Effects of cell phone radiofrequency signal exposure on brain glucose metabolism. JAMA 305: 808-813 [Crossref]

2. Dubey RB1, Hanmandlu M, Gupta SK (2010) Risk of brain tumours from wireless phone use. J Comput Assist Tomogr 34: 799-807 [Crossref]

3. Kleinlogel H, Dierks T, Koenig T, Lehmann H, Minder A (2008) Effects of weak mobile phone-electromagnetic fields (GSM, UMTS) on event related potentials and cognitive functions. Bioelectromagnetics. 29: 488-497 [Crossref]

4. Wainwright P1 (2000) Thermal effects of radiation from cellular telephones. Phys Med Biol 45: 2363-2372 [Crossref]

5. Logg C.R, Tai C.K, Log A, Anderson W.F, Kasahara N (2001) A uniquely stable replication-competent vector achieves efficient gene delivery in vitro and in solid tumours. Human Gene Therapy. 12: 921-932 [Crossref]

6. Benson V.S, Pirie K, Schuz J, Reeves G.K, Beral V, Green J (2013) Mobile phone use and risk of brain neoplasms and other cancers: prospective study. Int $J$ Epidemiol 42: 792-802 [Crossref]

7. Frei P, Poulsen A.H, Johansen C, Olsen J, Steding-Jessen M (2011) Use of mobile phones and risk of brain tumours: update of Danish cohort study. BMJ 11: 343

Copyright: $@ 2017$ Malikova MA. This is an open-access article distributed under the terms of the Creative Commons Attribution License, which permits unrestricted use, distribution, and reproduction in any medium, provided the original author and source are credited. 